\title{
Towards a Unification-Based Phonology
}

\section{Richard Wiese}

Seminar für Allgem. Sprachwissenschaft Heintich-Heine-Universität Düsseldorf D-4000 Düsseldorf 1 wieseœdd0rud81.bitnet

\section{Introduction: The Problem}

Phonological theory has undergone a number of more or less radical changes in recent years. Models of non-linear representation of features, constraints on the abstractness of rules, and theories of underspecification (imposing conditions on possible underlying features and their values, avoiding redundancy) are all part of current phonological thinking.

Considerations of the formalisms involved in these theories have not been pursued to a large extent. It has become increasingly clear, however, that different representational formats lead to differences in the formalisms required for phonological rules. Recently, there have also been attempts to clarify the formal status of associations between elements on different tiers (Sagey 1988, Hammond 1998, Bird \& K1ein 1989). This paper is an attempt to clarify some of the properties of phonological tuies and representations by applying the concepts of unification formalisms to phonological theory.

A crucial part of any unification-based phonological theory is the elimination of feature-changing rules from the list of possible rules in the framework. Feature-changing rules stand in clear opposition to the incremental, monotonic nature of unification grammars. In the following, it will be shown how a change of feature-values can be avoided for a rule which, first, is a regular phonological rule within any phonological theory that allows for phonological rules at all, and which, second, has always been formulated as a featurechanging rule (see literature from Kiparsky 1968 to Rubach 1990). ${ }^{1}$

\section{A standard analysis of German Final Devoicing}

The rule in question is one of the classical examples of a neutralization rule, namely the rule of Final Devoicing (Auslautverhärtung, FD for short) in the phonology of Standard German. It neutralizes the phonemic contrast between voiced and unvoiced obstruents in a particular position (namely
Institut fir maschinelle

Sprachverarbeitung

Universität Stuttgart

D-7000 Stuttgart 1

syllable-finally). The standard view of this rule is that it changes voiced obstruents in syllable-final position into their unvoiced counterparts. Examples are provided in (1), and a feature-changing version of this rule is given in (2). It is clear now that the relevant domain for the rule is the syllable (see Vennemann 1978, Wiese 1988); depending on the model of syllabic structure, the precise formulation of the context description can vary, referring to the syllable edge as in (2) or to the final constituent of the syllable. ${ }^{2}$

(1)

Lo[p] - Lo[b]es 'praise (nom.) - 'praise (gen.)'

Ra[t] - Ra[d]es 'wheel (nom.)' - 'wheel (gen.)'

Sar[k] - Sär[g]e 'coffin (sg.)' - 'coffin (p1.)'

akti[f] - akti[v]e 'active' - 'active (infl.)'

Gra[s] - Grä[z]er 'grass (sg.)' - 'grass (p1.)'

oran[S] - Oran[z]e 'orange (colour)'

- 'orange (fruit)'

(2) Finat Devoicing:

$[$ - sonorant $\rightarrow[$ - voiced] $/[$ syllable

In (3), the application of the rule (2) is exemplified. Note that the rule changes the value of a feature. In the framework assumed, this is necessarily so, since in the [- sonorant]-class, voicing is distinctive, so that both $/ b /$ and $/ p /$ are assigned this feature. (4) demonstrates that a different syllabification (due to suffixation) correctly prevents FD from applying. ${ }^{3}$
(3)

a. /lo:b/

b. $-\cdots$

c.
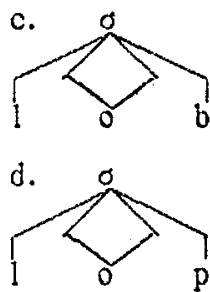

(4)

a. /lo:b/

b. $/ 10: b+2 s /$ syllabification

$\mathrm{FD}(2)$ 


\section{The alternative solution}

How could FD be formulated in a non-featurechanging way without giving up the goal of capturing all generalizations that hold for this rule? The following considerations rely on some (fairly uncontroversial) assumptions about phonological representation. First, in accordance with underspecification theory, it will be assumed that redundant feature values are not present in underlying representations (lexical entries). For the relevant segments this means that the voiced obstruents are marked as [+ voiced], whereas unvoiced obstruents are not marked for this feature. General considerations of markedness support this choice: Voiceless obstruents are more common, have a wider distribution, are acquired earlier in language acquisition, etc.

Secondly, all features, including those representing a segment, are represented in a non-linear, associative way. In particular, a hierarchical model of the segment in the version of MoCarthy (1988:105) is adopted. In this particular version of a feature hierarchy, all nodes are characterized by the presence of particular features that constitute the node. (5) is not quite complete, but allows the re- presentation of most natural classes of segments. ${ }^{4}$ (Conceivably, as argued by Goldsmith (1990: ch. 6.2 ), under assumptions of underspecification, the feature hierarchy in (5) and in comparable proposals is too elaborate. For the present purposes, the only important assumption is that features and their values are organized hierarchically.)

We will interpret the feature hierarchy in (5) as an admissibility tree. Features are only allowed if they enter the relations expressed in this tree. Furthermore, the relations ("associations" in the terminology of non-linear phonology) are interpreted as being transitive. The values of features are propagated (as instructions to the articulatory apparatus) along the paths specified by the relations to the root. In (6), the feature tree (5) is recoded into a complex feature structure. Every feature takes as a value either one of the terminals ("+" or "-") or another feature structure. Associations are expressed by a feature $A$ with the interpretation for the associations as just given. (Different instances receive numerical indices.) As a template for possible segments, this structure does not contain the terminal values, i.e. + and -. These values are denoted by '[]', the empty feature structure, if unspecified.

(5) A hierarchy for segmental features:

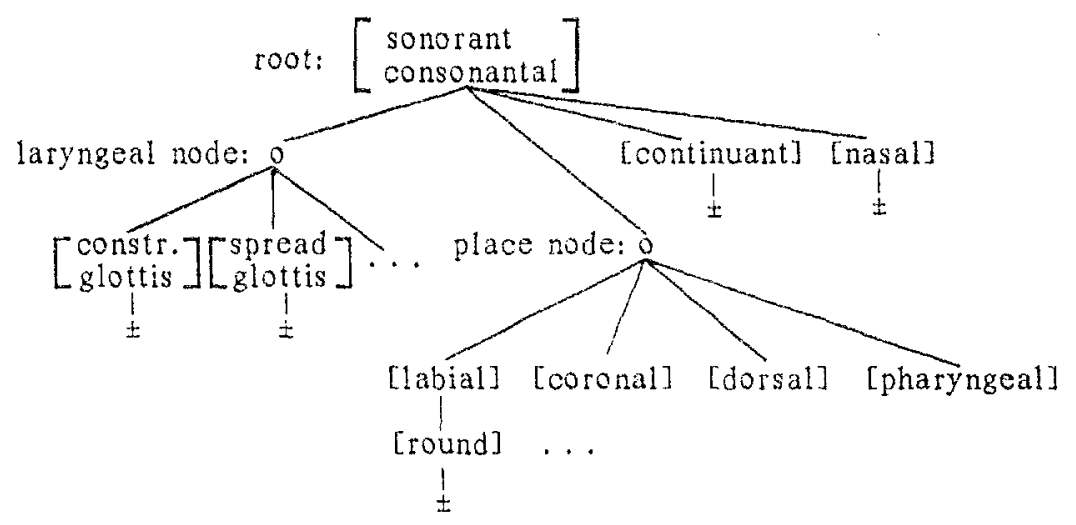

(6)

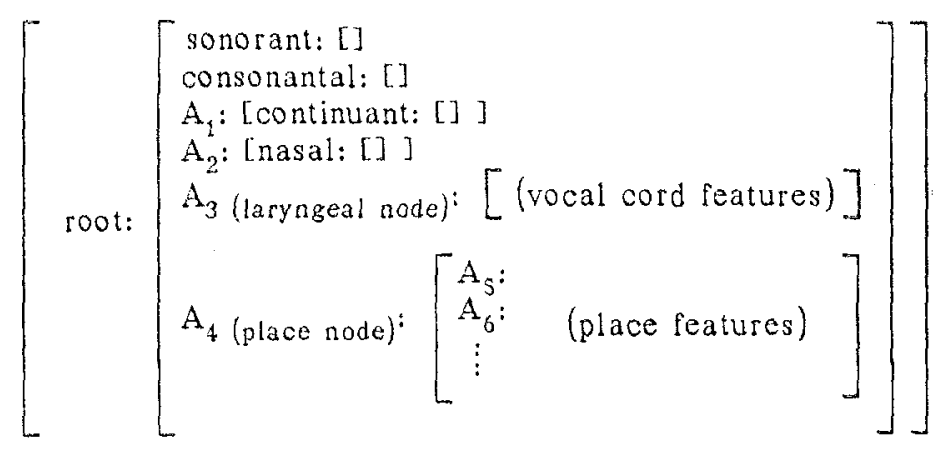


Operations on feature trees or structures of this kind are very limited. We allow only the follo wing types of formal operations:

- insertion of associations, ${ }^{5}$

- deletion of associations ("delinking").

The first operation generally occurs through unification. Unification here is the combination of lexical entries and regularities that are independently motivated and expressed as feature structures. The information in the feature structures is combined, leading to statements that particular relations hold. In particular, the assignment of default values for features is achieved through unifying the feature structures that express the default value with representations for lexical entries that do not contain a specified value for the respective feature. Dolinking, on the other hand, is not so readily modeled with unificational means (as presented by Shieber 1986 or Carlson \& Linden 1987); it can be seen, however, as the introduction of generalization ("the dual of unification", Shieber (1986: 64)) into the theory. Undet generalization of two feature strucures, only information contained in both is kept; all other information is lost. We will now show that using generalization alongside unification allows us to state FD without the invocation of a feature-changing rule.

The typology of rules proposed here does not allow feature values to be changed. The treatment of FD in German, then, is the following. Taking / $/$ / and $/ t /$ as examples, marking for [voiced] is expressed underlyingly as in (7). ${ }^{6}$ There exists a default rule (8) that in general (i. e., in the absence of other provisions) allows values for [voiced] to be identical to the value for the feature [sonorant]. This rule uses feature reentrancy. (Possibly, sonorancy itself is also underspecified, but this is left out of consideration here.) Furthermore, there is no rule of FD but a well-formedness condition (9) which explicitly requires obstruents to be [- voiced] syllable-finally. (8) and (9) are short-hand notations for the more complex formulations within the feature structure. The crucial step in the derivation of, say, Ra[t], with an underlying $/ d /$, is the generalization of the two feature structures (7a) and (9), with different values for [yoiced]. As a result, [voiced] is unspecified. (10) illustrates the steps in the application of the various mechanisms leading to the devoicing of $/ d /$. The apparent change in the value vor [roiced] is possible because of generalization, which removes a value, and the existence of the default rule, which provides a value.

(7)
a. $/ d /$ : [voiced: + ]
b. $/ t /$ : [voiced: [] ]

(8)

[ sonorant: (1) voiced: (1)

(9)

(10)
a. $\left[\begin{array}{l}\text { sonorant: } \\ \text { voiced: }\end{array}\right.$
underlying
b. $\left.\left[\begin{array}{l}\text { sonorant: } \\ \text { voiced: }[]\end{array}\right]\right]_{\text {syllable }}$
syllabification, generalization with (9)

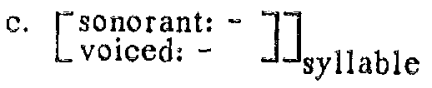
default $\sim$ rule, unification with (8)

It must be stressed that this proposal for the treatment of FD has one major advantage over the traditional analysis: While it is clear that neutralization processes such as FD always disallow the more marked member of the pair in question, there is nothing in the formalism for the FD rule (2) that would formally disallow a tule that leads to the marked member of the pair. This, however, is impossible in the framework introduced here. If a wellformedness condition analogous to (9) would require a voiced obstruent syllable-finally, there would not be a rule which could provide the value [t voiced]. There simply is no rule comparable to default rule (8). In other words, neutralization for [voiced] can only lead to [- voiced] because there is a well-motivated default rule providing this value. In this sense, the solution in the framework given has a higher explanatory level than the ones operating with a feature-changing rule, where both types of change, from marked to unmarked and from unmarked to marked, are equally complex.

\section{Conclusions}

The relationship between phonology and computational linguistics has various aspects. On one level, one might want to argue for a better treatment of phonological knowledge in language-processing systems (see remarks in Wiese 1986). On another level, it is instructive to observe how phonological theory can be shaped by explicitly computational considerations.

Rules in non-linear phonology are to a large extent not feature-changing, especially if underspeci- 
fication theory is utilized as well. This observation constitutes additional motivation for the line of reasoning in this paper. We hope to have shown, first, that an analysis of $F D$ without the invocation of a feature-changing rule is actually the preferred solution. Secondly, a large number of well-established rules, namely the members of the class of neutralization rules, can be analysed as particular instances of unification.

However, the concept of generalization (as a formal interpretation of delinking as this concept is standardly used in non-linear phonology) cannot be avoided in an adequate treatment of these cases. It is in the very nature of neutralization that some information (here on the voicing of a consonant) is not retained under specific circumstances. In that sense, phonological rule applications cannot constitute a strictly monotonic system, unless we are willing to give up well-motivated linguistic generalizations. Finally, we take side with Hammond (1988) on the question of how association lines are to be interpreted: transitivity is a crucial property of these relations, since the feature structures are highly hierarchical.

Future work in this area must also answer the question what principles determine and restrict the applicability of generalization. Generalization should not be applicable whenever unification fails. But it seems to be in the nature of phonology that linguistic items can be realized even if they violate some valid wellformedness conditions: Lexical entries in German end up being realized as words despite the fact that they contain an underlying voiced obstruent which violates FD. In numerous other cases, whole segments are deleted because they do not conform to phonotactic patterns or similar constraints. It is in this area that unification must be supplemented with generalization.

\section{Footnotes}

1) Theories with only one level of description, especially in the work of Vennemann, provide the major exception to this statement.

2) Application of FD to recent loan words such as orange in (1) shows first that this rule is totally productive, and, secondly, that it is indeed applicable to all segments marked as [- sonorant]. The native stock of German words does not display a phoneme $/ 3 /$, but in orange this sound is readily devoiced to $/ S \%$.
3) Details of syllable structure are omitted, because they are irrelevant for present purposes.

4) The feature [voiced] used here in the description of FD is probably a short-hand notation for a particular configuration of the laryngeal states. In (5) it is expressed as a particular configuration of glottal states.

5) This comes in two sub-cases: the spreading of a feature value to several nodes, as in the various harmony and assimilation rules, and the linking of a "floating" feature to a node.

6) The markings in (7) hold for all occurrences of $/ d /$ and $/ t /$. Here lies the difference to the classical archiphonemic treatment of neutralization (Jakobson 1929, Trubetzkoy 1939), where an archiphoneme $/ D /$ is postulated only in the context of neutralization.

\section{Reference:}

Bird, S. \& E. Klein (1989) Phonological Events. Ms.. University of Edinburgh.

Carlson, L. \& K. Linden (1987) Unification as a Grammatical Tool. Nordic Journal of Linguistics 10. 111-136.

Goldsmith, J. A. (1990) Autosegmental and Metrical Phonology. Oxford: Blackwell.

Hammond, M. (1988) On Deriving the Well-Formedness Condition. Linguistic Inquiry 19, 319-325.

Jakobson, R. (1929) Remarques sur l'Evolution phonologigue du russe. Prag: TCLP 2. (Reprinted in: R. Jakobson (1962) Selected Writings I. The Hague: Mouton.)

Kiparsky, P. (1968) Linguistic Universals and Linguistic Change. In: E. Bach/R.T. Harms (eds.): Universals in Linguistic Theory. New York et al: Holt, Rinehart and Winston, 170-202.

McCarthy, J. J. (1988) Feature Geometry and Dependency: A Review, Phonetica 43, 84-108.

Rubach. J. (1990) Final Devoicing and Cyclio Syllabification in German. Linguistic Inquiry 21, 79-94.

Sagey, E. (1988) On the III-Formedness of Crossing Association Lines. Linguistic Inquiry 19, 109-118.

Shieber. S. M. (1986) An Introduction to Unification-Based Approaches to Grammar. Stanford: Center for the Study of Language and Information.

Trubetzkoy, N. S. (1939) Grundzigge der Phonologie. Prag: TCLP 7

Vennemann, T. (1978) Universal Syllabio Phonology. Theoretical Linguistics 5, 175-215.

Wiese, R. (1986) The Role of Phonology in Speech Processing. In: Proceedings of COLING 86. Institut fur Kommunikationsforschung und Phonetik: Bonn, 608-611.

Wiese, R. (1988) Silbische und lexikalische Phonologie. Studien zum Chinesischen und Deutschen. Tubingen; Niemeyer. 